\title{
An Antimicrobial Efficacy of Different Hand Sanitizers
}

\author{
Hayyan Ismaeil Al Taweil ${ }^{*} \quad$ Yahya. S. Al Dawood \\ Department of Clinical Laboratory Science. Mohammed Al-Mana College for Medical Sciences. Al-Khobar, \\ Eastern Province, Kingdom of Saudi Arabia, PO Box3915- Zip Code 34222
}

The research is financed and supported by Research Unit in Mohammed Al-Mana College for Medical Sciences. Ethical approval number ( Scientific Research Unit is pleased to inform you that your proposal entitled "An antimicrobial efficacy of different hand sanitizers " is approved from IRB with the approval number "SR/RP/46". Abstract

Hand hygiene, hand sanitizing, is essential in reducing the transmission of infectious disease.The outbreaks of any infectious diseases increased public awareness of the practice of hand sanitizing and resulted in the introduction of new products to the markets. This study assesses the antibacterial activity of selectedhand sanitizer products against some of the clinical isolates using diffusion susceptibility tests methods. The mean diameters of zones of inhibition (in mm) observed in Group 1, Group 2, Group 3, Group 4 and Group 5 were (21.4 \pm 6$),(08.0 \pm 0.5),(11.9 \pm 1.5),(11.4 \pm 0.5)$ and $(20.6 \pm 1)$, respectively. Maximum inhibition was found with Group 1 against all the tested organisms. Data were statistically analyzed using analysis of variance and also were statistically significant at $P<0.001$. Results showed higher inhibitory activity of the products to Staphylococcus aureusthan Escherichia coli and Pseudomonas AeruginosaAll tested sanitizers showed higher zones of inhibition, indicating their overall effectiveness.

Keywords: Anti microbial agent, hand sanitizers, hygiene, organisms.

DOI: $10.7176 / \mathrm{JBAH} / 11-14-03$

Publication date:July $31^{\text {st }} 2021$

\section{1- Introduction}

Hospital and community- acquired infections areconsidered a serious public health problemworldwide (Hassan et al., 2012). Personal as well as hand hygiene is important to prevent many communicable diseases. As transmitting modes of microbes, hands are considered to be the primaryroute for the causative microbes and infections to the individuals (Modal et al., 2004).

The Centers forDisease Control and Prevention, the World HealthOrganization, promotehand hygiene as the most important measurein the prevention of hospital and community- acquired infections (World Health Organization, 2009; Pittet D, et al., 2009; Madan K, et al., 2012). Applying both washing hands with soap and hand antiseptics are the most important hygiene methods which significantly increase the chance ofmaintaining the hands clean and aseptic.Staphylococcus aureus, Staphylococcus epidermidis, and Enterococcusfaecalis conserved as resident floras and commonly colonized the different place of body such as superficial layers ofskin that consists of S. aureus, Escherichia coli, and Pseudomonas aeruginosa (Widmer AF, 2003).

It was explained well that handwashing removes body's own fatty acids from theskin, which may cause cracked skin that formedan entry portal for pathogens, using of hand washing, hand sanitizerswere introduced to prevent those pathogenic micro-organisms as well as toprotect and improve skin condition (Winnefeld M, et al., 2000; Bloomfield SF, 2007). Furthermore, toreduce infections in the community, alcohol-basedhand sanitizers are recommended as a component of hand hygiene (Jain VM, et al., 2016). Hand hygiene generally refers to different methods of eliminating or killing microorganisms which may be present on hands, by either hand washing or sanitizing (Pittetet al., 2006; Zapkaet al., 2017). Though the concept of hand sanitization has been in place right from the start of the hand hygiene campaign by Semmelweis (WHO 2009; Pires et al., 2017), many hand sanitizers are available in the marketwith different effectivenessagainst pathogenic microbes some of themin the market as antimicrobialhand sanitizers are not effective in reducing bacterialcounts on hands. In fact, despite a label them ofreducing "germs and pathogenic bacteria" by $99.9 \%$, some studies have observed an apparent increase inthe concentration of bacteria in handprints impressedon agar plates after cleansing (Reynolds SA, et al., 2006). Hence, there still needto verify these claims and to overcome this issue, the present study was carried out to assess theantimicrobial effectiveness of four different handsanitizers against sometested organisms.Most of the early reports focusedon the role of hand washing as an infection control measure (Garner and Favero 1986). This changed by 2000s, when the Centers for Disease Control and Prevention (CDC) issued a guideline recommending that alcohol-based hand rub (ABHR) be routinely used for decontaminating hands which are often composed of alcohol, ethanol, isopropanol or propanol (Pittet 2001; Pickering et al., 2010).It has been recommended that concentration range of $60 \%$ to $95 \%$ (Reynolds et al., 2006). The use of alcohol-based hand sanitizers has been reported as one of the commonly recommended means of hand hygiene for outbreaks of the disease (Wolfe et al., 2017), particularly for hands that are not visibly soiled. These hand sanitizers have been shown to be effective in various situations such as the reduction of gastrointestinal infection, reducing infection 
(Meadows and Le Saux 2004; Reynolds et al., 2006) and has been reported to give better results than hand washing (Pickering et al., 2010). Scientific studies have shown that after hand washing, as many as $80 \%$ of individuals retain some pathogenic bacteria on their hands (Tambekar DH, et al, 2007). The outbreak of diseases led to an increased awareness of the role of hand sanitizers in infection control (Olalekan and Adeola 2014; Nwabuezeet al., 2016).Most of these products have made numerous claims, notably their ability to eliminate 99.9\% of microorganisms (Odebisi-Omokanye 2015). Therefore, pathogenic organisms were selected to assessment the antimicrobial efficacy of different hand sanitizers.

\section{2- Material and Methods}

The present study is an in vitro study conducted at the Department of Clinical Laboratory Science- Mohammad Almanaa College. Ethical clearance for the study was obtained from the scientific research unit.

2.1- Different brands of hand sanitizers were selected out of many available in the market based on their popularity usage.

- Group 1: Propan-2-ol, Propan-1-ol, Mecetronium ethyl sulfate, Glycerol, Tetradecane-1-ol, fragrances, Patent blue V, Purified water

- Group 2: Hrivera, Coriander, Lime, Ushira, Neem

- Group 3: Denatured Alcohol- 69.4\% w/w, Water PEG/PPG-17/6 copolymer, Propylene glycol, Acrylate/C10-30 alkyl acrylate, cross polymer, Tetrahydroxpropyl ethylenediamine, Perfume.

- Group 4: 95\% v/v IP 55\% w/w, Isopropyl alcohol 10\% IP w/w, Tocopheryl acetate IP 0.0

- Group 5 Ideal: 70\%isopropyl alcohol, 0.5\%cholorohexidine, emollients

- Group 6 Sterilized Distilled Water -SDW as negative control.

\section{2- Tested microorganisms}

Mueller-Hinton, nutrient broth agar and nutrient agar were used for agar diffusion for bacterial isolate preservation. The clinical isolates of Staphylococcus aureus,-, Escherichia coli and Psuedomonasaeruginosa were obtained from the culture plates of the respective microorganisms preserved on the nutrient agar slants and were stored at $4^{\circ} \mathrm{C}$ in the laboratory of Microbiology in Clinical Laboratory Science Department.

\section{3- Susceptibility test}

Disk agar diffusion technique was used for the evaluationof antimicrobial efficacy of hand sanitizers using previously described methods (Otokunefor and Dappa 2017; Magaldi et al., 2004; CLSI, 2012). The agar well diffusion testwas carried out as a preliminary screen to assess the antimicrobial activities of the various products. This involved the use of an inoculum corresponding to $0.5 \mathrm{McF}$ arland.

2.4- Minimum Inhibitory Concentration (MIC): MIC testing was carried out to determine the minimum concentration of test substances which could cause an inhibition of the growth of the test isolates. Following 24 hours incubation at $37^{\circ} \mathrm{C}$, the MIC was determined as the lowest concentration of test substance which caused an inhibition of the growth of the test organisms. The test inoculums was swab inoculated to a Mueller Hinton agar plate and allowed to stand at room temperature for 15 minutes. Following this, 4 wells were created on the plates using a $6 \mathrm{~mm}$ cork borer and $0.2 \mathrm{ml}$ of differing concentrations $(100 \%, 50 \%$ and $25 \%)$ with three replicates of the test substance added to individual wells. After 24 hoursincubation at $37^{\circ} \mathrm{C}$, the zones of inhibition were then measured (Otokunefor and Dappa 2017).

2.5- Minimum Bactericidal Concentration (MBC): Todetermine the $\mathrm{MBC}$ of each test substrate, against each test isolate, the three lowest concentrations which resulted in an inhibition of the test organism were subcultured into nutrient agar plates, incubated at $37^{\circ} \mathrm{C}$ for 24 hours and observed for growth. The MBC was taken as the least concentration which did not result in the growth of the organism (Otokunefor and Dappa 2017).

\section{6- Statistical analysis}

Data were statistically analyzed with analysis ofvariance followed by post hoc test for group-wisecomparisons. All statistical procedures were performedusing Statistical Package for Social Sciences (SPSS) version 21.0 software (IBM, Armonk, NY, USA). zone of inhibition (in mm) wasanalyzed using the mean of all the readings obtained,and the difference in the values of different handsanitizers was statistically significant at $P<0.001$.

\section{3- Results and Discussion}

All tested organisms were affected by Hand sanitizers. the zone of inhibition against the particular test organismwas performed to assess the antimicrobial effectiveness was assessed by measuring. Maximum inhibition (in $\mathrm{mm}$ ) was seen in Group 1 i.e., $21.4 \pm 1.414$ and minimum in Group 2 i.e., $8 \pm 0.95$. The difference 
in the values of the different sanitizers was statistically significant $[P<0.001$, Table 1 .

Group 1 showed the highest antimicrobial effectiveness followed by Group 2, 3, 4and Group 5, against all the different test organisms used in the study. Group $1 \& 5$ could inhibit all of the tested bacteria, whereas other group of sanitizers showed a limited action (Table 1).

It was found statistically high significant difference was observed against all the bacterial isolates when Group 1 was compared with any other group. However, there was no statistically significant difference when Group 2, Group3, and Group 4 were compared with each other against all test organisms. The mean difference is statistically significant at the 0.05 level. The highest inhibition zoon was register for group 1 against $S$. aureus $(24.9 \mathrm{~mm})$ while the lowest was for group 2 against $E$. coli $(6.6 \mathrm{~mm})($ Table 1,2$)$

All hand wash products exhibited inhibitory activity against the test isolates (Table 2), with zones of inhibition ranging from $14.2 \mathrm{~mm}$ to $24.2 \mathrm{~mm}$ at concentrations of $100 \%$, while it was ranging from (6.0 to 16.2) $\mathrm{mm}$ at concentrations of 50\%. A general reduction in inhibitory activity was associated with a reduction in product concentration, and inhibition was still observed at concentrations as low as $25 \%$, in some cases (Table 2). The widest variation was observed with group 1 and 5 which showed a high \% inhibition in growth of against all tested microbes as $8-24.2 \mathrm{~mm}$ inhibition of $S$. aureus growth. Group 2 was the least effective. This product showed low activity at all against al microbes comparing to others. An MIC of $25 \%$ (Table 2), Group 1 and 5 appeared to be the more effective hand wash as it showed an MIC less than $50 \%$ in $75 \%$ of cases as opposed to the other products which showed an MIC of $>50 \%$ only in $50 \%$ of cases (Table 3).while MBC was varied among microbes and groups and ranged from $25-100 \%$ asshowed in table 3 .

\section{4- Discussion}

The Human body and especially normal human skin always harbor bacteria $\left(10^{2}\right.$ and $\left.10^{6} \mathrm{CFU} / \mathrm{cm}^{2}\right)$. As main source of transfer infections, hand considered to play main role in transfer of bacteria from the hands to food, so that people plays an important role in the spread of many communicable diseases. (Kimura AC, et al., 2004). Microbe transferring from hands and causing infections, depends on many factors such as; density of microorganisms on the hands, the type and duration of contact, the type of micro-organism, resident flora, and their colonization resistance (Mondal S, and Kolhapure SA. 2004

Table 1: Zone of inhibition as antimicrobial efficacy of different hand sanitizers. Labeling on the side of respective zone of inhibition as groups.

\begin{tabular}{lllllll}
\hline Test Organism & \multicolumn{7}{c}{ Zone of inhibition (in mm) } \\
& Group 1 & Group 2 & Group 3 & Group 4 & Group 5 & Group 6 \\
S. aureus & 24.9 & 9.2 & 12.8 & 12.2 & 21.5 & 6.0 \\
E. coli & 20.2 & 6.6 & 13.6 & 13.6 & 19.2 & 6.2 \\
$\boldsymbol{P}$. aeruginosa & 19.8 & 8.2 & 9.2 & 8.5 & 21.2 & 6.5 \\
Range & 21.4 & 8 & 11.9 & 11.4 & 20.6 & 6.0 \\
\hline
\end{tabular}

The difference in the values of different hand sanitizers was statistically significant at $P<0.001$.

To overcome the negative impact of microbial contamination, hand sanitizers are recommended as a hygiene technique to keep hands clean. (Boyce JM, and Pittet D, 2002)

Most commonly and available hand sanitizers in the market were selected for the study. Among the four hand sanitizers used in this study, group 1, 3, 4 and, 5 were alcoholbased and group 2 was herbal, i.e., nonalcohol-based hand sanitizer. Many studies have been conducted to assess the antimicrobial effectiveness of hand sanitizers alone, but very few literatures are available to assess the difference between various disinfectants and hand sanitizers. Disinfectants are chemical agents with an immediate and sustained activity which destroys micro-organisms to such a level mandated for hygienic and surgical indications. On the other, handsanitizers are agents with an activity to reduce micro-organismsnumbers meet the public health requirements. Alcohol is the better form as disinfectant (propanol) to reduce bacteria as compared to ethanol sanitizers in 15-30 s.

One of the previous research studied the impact of hand hygiene in disease prevention, they found that hand sanitizing has more recently been the proscribed method of hygiene, possibly due to the higher compliance rates associated with it and its particular usefulness in areas lacking adequate water supply (Nwabuezeet al., 2016). Over the years, $S$. aureus and E. coli have been documented as the two most common pathogens isolated in the clinical microbiology laboratory. These organisms are notorious for their ability to cause a wide variety of diseases, exhibit a wide repertoire of virulence factors and a high level of antibiotic resistance. Additionally, along with K.pneumoniae and P. aeruginosa, these organisms canbe spread via the hands. This study found variable efficacy of the hand sanitizers assessed. While similar levels of inhibition were noted against S. aureus and K.pneumonia. Based on the results, the variable level of activity of hand sanitizers were reported, by Sharif and Ansari, and found that the efficacy of many types hand sanitizing, that only one product was effective against $6.5 \%$ of the isolates tested (Sharif and Ansari 2015). 
Table 2: Inhibitory effect of hand sanitizers detected by Agar well diffusion technique.

\begin{tabular}{|c|c|c|c|}
\hline \multirow[t]{2}{*}{ Antibacterial Agent } & \multicolumn{3}{|c|}{ Zone of Inhibition (mm) } \\
\hline & S. aureus & Escherichia coli & Pseud \\
\hline \multicolumn{4}{|l|}{ Group 1} \\
\hline $100 \%$ & 24.2 & 20.1 & 18.2 \\
\hline $50 \%$ & 24.0 & 19.2 & 12.5 \\
\hline $25 \%$ & 15.2 & 16.2 & 7.8 \\
\hline NC & 7.0 & 5.5 & 5.0 \\
\hline \multicolumn{4}{|l|}{ Group 2} \\
\hline $100 \%$ & 14.5 & 14.2 & 16 \\
\hline $50 \%$ & 9.2 & 9 & 7 \\
\hline $25 \%$ & 5.2 & 7 & 7 \\
\hline NC & 6.0 & 7 & 7 \\
\hline \multicolumn{4}{|l|}{ Group 3} \\
\hline $100 \%$ & 18 & 19.2 & 20.2 \\
\hline $50 \%$ & 16 & 6.5 & 6 \\
\hline $25 \%$ & 8.2 & 6 & 6 \\
\hline NC & 8 & 6 & 6 \\
\hline \multicolumn{4}{|l|}{ Group 4} \\
\hline $100 \%$ & 15.2 & 16 & 20 \\
\hline $50 \%$ & 10.0 & 6 & 6 \\
\hline $25 \%$ & 8 & 6 & 6 \\
\hline NC & 8 & 6 & 6 \\
\hline \multicolumn{4}{|l|}{ Group 5} \\
\hline $100 \%$ & 20.2 & 18 & 18.2 \\
\hline $50 \%$ & 14.9 & 17.5 & 17 \\
\hline $25 \%$ & 10 & 7.0 & 7 \\
\hline NC & 8 & 7.0 & 7 \\
\hline
\end{tabular}

The difference in the values of different hand sanitizers was statistically significant at $P<0.001$.

Furthermore, other study by Ochwotoet al, (2017) found that $25 \%$ of tested products were effective against only $33 \%$ of the test isolates. While study reported a possible link of efficacy to composition and noted that the ethanol-based products resulted in a higher efficacy than the isopropyl based products.For most alcohol-based hand sanitizers, the alcohol components are the major active ingredients. These act by disrupting tissue membranes, denaturing proteins and dissolving lipids (Oke et al., 2013). It therefore follows that products with higher alcohol concentrations (up to 90\%) would be more effective than products with lower alcohol concentrations (below 60\%). Similar to a previous report (Odebisi-Omokanyeetal., 2015), our study noteda lower level ofsusceptibility to all the tested products in 2 of the 3 Gram negative organisms tested. Both $S$. aureus and $K$. pneumoniae however, showed similar levels ofsusceptibility. The results of this study were totally agreed with both (Oke et al., 2013; Odebisi-Omokanye et al., 2015). And unlike both studies which reported a total lack of bacteriocidal activity possibly due to improper storage, all hand sanitizing products in this study exhibited bactericidal activity this point at the general effectiveness of the products assayed in this study.

Table 3: MIC of hand sanitizers against tested isolates

Sanitizer

Group
Tested microbes

Escherichia coli

MIC $\quad$ MBC

$25 \%$

$50 \%$

$100 \%$

$100 \%$

$100 \%$

$50 \% \quad 25 \%$

$25 \%$

\section{Pseudomonas Aeruginosa}

$\begin{array}{ll}\text { MIC } & \text { MBC } \\ 50 \% & 100 \% \\ 50 \% & 100 \% \\ 50 \% & 100 \% \\ 50 \% & 100 \%\end{array}$

Group $5 \quad 25 \% \quad 50 \%$

The difference in the values of different hand sanitizers was statistically significant at $P<0.001$. 
5- Conclusion: While the results of this study show thatthe products assayed have higher efficacy and higher inhibitory activity to S. aureus than E. coli andP.Aeruginosa. All the sanitizers showed good activities, with inhibition of bacteria noted at concentrations as low as $25 \%$ and $\mathrm{MBC}$ was varied among microbes and groups and ranged from $25-100 \%$. More stringent checks of products introduced into the market may therefore be necessary to ensure that they meet set international standards both in the composition of inhibitory substance and texture to ensure uniformity in activity against pathogens.

\section{6- AKNOWLEDGMENT}

Research stared by the supporting of Research Unit in Mohammed Al-Mana College for Medical Sciences. Ethical approval number ( Scientific Research Unit is pleased to inform you that your proposal entitled " An in vitro assessment of antimicrobial efficacy of different hand sanitizers " is approved from IRB with the approval number "SR/RP/46".

\section{7- References}

1. Bloomfield SF (2007). The effectiveness of hand hygiene procedures in reducing the risks of infections in home and community settings including handwashing and alcohol-based hand sanitizers. Am J Infect Control; 35:27-64.

2. Boyce JM, \& Pittet D (2002). Healthcare Infection Control Practices Advisory Committee; HICPAC/SHEA/APIC/IDSA Hand Hygiene Task Force. Guideline for Hand Hygiene in Health-Care Settings. Recommendations of the Healthcare Infection Control Practices Advisory Committee and the HICPAC/SHEA/APIC/ IDSA Hand Hygiene Task Force. Society for Healthcare Epidemiology of America/Association for Professionals in Infection Control/Infectious Diseases Society of America. MMWR Recomm Rep 2002;51:1-45

3. Centers for Disease Control and Prevention (2002). Guideline for hand hygiene in health-care settings. Recommendation of the Healthcare Infection Control Practices Advisory Committee and the HICPAC/SHEA/APIC/IDSA Hand Hygiene Task Force.

4. Garner, JS; Favero, MS (1986). Guideline for handwashing and hospital environmental control, 1985 supersedes guideline for hospital environmental control published in 1981.

5. Hassan AO, et al (2012). A survey of Enterobacteriaceae in hospital and community acquired infections among adults in a tertiary health institution in Southwestern Nigeria. Afr J Microbiol Res; 6:5162-7.

6. Jain VM, et al (2016). Comparative assessment of antimicrobial efficacy of different hand sanitizers: An in vitro study. Dent Res J;13:424-31

7. Kimura AC, et al (2004). Multistate shigellosis outbreak and commercially prepared food, United States. Emerg Infect Dis; 10:1147-9.

8. Madan K, et al (2012). Comparative evaluation of efficacy of alcoholic vs. non-alcoholic hand sanitizers. Int J Life Sci Biotechnol Pharma Res; 1: 173-7.

9. Magaldi, S; et al (2004). Well diffusion for antifungal susceptibility testing. International Journal ofInfectious Diseases. 8(1), 39 - 45.

10. Meadows, E; \& Le Saux, N (2004). A systematic review of the effectiveness of antimicrobial rinse-free hand sanitizers for prevention of illness-related absenteeism in elementary school children. BMCPublic Health. 4(1): 50.

11. Mondal S, \& Kolhapure SA (2004). Evaluation of the antimicrobial efficacy and safety of pure hands herbal hand sanitizer in hand hygiene and on inanimate objects. Antiseptic; 101:55-7.

12. Nwabueze, SA; at al (2016). Ebola viral disease prevention: Perception of secondary school students in two districts in Anambra State, Nigeria. Issues inScientific Research. 1(1): 1 - 9.

13. Ochwoto, M; et al (2017). Anti-bacterial efficacy of alcoholic hand rubs in the Kenyan market, 2015. Antimicrobial Resistance \& Infection Control. 6 (1): 17.

14. Odebisi-Omokanye, MB; et al (2015). Comparative Assessment of Antibacterial Efficacy of four popular hand sanitizers sold in Nigeria. FountainJournal of Natural and Applied Sciences. 4(1): 1- 9.

15. Oke, MA, at al (2013). Evaluation of antibacterial efficacy of some alcohol-based hand sanitizers sold in Ilorin (North-Central Nigeria). Ife Journalof Science. 15(1): 111 - 117.

16. Olalekan, AW, \& Adeola, E (2014). How prepared are Nigerian schools for ebola virus disease prevention and control? Annals of Global Health. 80(6): 452 - 457.

17. Otokunefor, K, \& Dappa, B (2017). Antibacterial Evaluation of Nigerian Ocimum Sanctum Leaf Extracts against Bacterial Isolates Associated With Urinary Tract Infection. Nigerian Journalof Pharmaceutical andAppliedScienceResearch. 6(1): 19-25.

18. Pickering, A, et al ( 2010). Efficacy of waterless hand hygiene compared with handwashing with soap: a field study in Dar es Salaam, Tanzania. American Journal of Tropical Medicine and Hygiene 82 (2), 270- 
278.

19. Pires, D, et al (2017). Why language matters: a tour through hand hygiene literature. Antimicrobial Resistance\& Infection Control. 6(1): 65.

20. Pittet D, et al (2009). The World Health Organization guidelines on hand hygiene in health care and their consensus recommendations. Infect Control Hosp Epidemiol; 30:611-22.

21. Pratt RJ, \& Pellowe C (2001). The epic project: Developing national evidence-based guidelines for preventing healthcare associated infections. Phase 1: Guidelines for preventing hospital-acquired infections. Department of Health (England). J Hosp Infect 2001; 47 Suppl 1:S3-82.

22. Reynolds, et al (2006). Hand sanitizer alert. Emerging Infectious Diseases. 12(3): 527 - 529.

23. Sharif, M;\& Ansari, F (2015). Hand Sanitizers: Efficiency against fromCurrency Notes and Coins in Local Circulation. PakistanJournal of Molecular Medicine. 2(2): 7

24. Tambekar $\mathrm{DH}$, et al (2007). Prevention of transmission of infectious disease: Studies on hand hygiene in health-care among students. Cont J Biomed Sci;1:6-10

25. Widmer AF (2010) Replace hand washing with use of a waterless alcohol hand rub? Clin Infect Dis;31:13643

26. Winnefeld $\mathrm{M}$, et al (2000). Skin tolerance and effectiveness of two hand decontamination procedures in everyday hospital use. Br J Dermatol; 143:546-50.

27. Wolfe MK, (2010). Handwashing and Ebola virus disease outbreaks: A randomized comparison of soap, hand sanitizer, and $0.05 \%$ chlorine solutions on the inactivation and removal of model organisms Phi6 and E. coli from hands and persistence in rinse water https://journals.plos.org/plosone/article?id=10.1371/journal.pone. 0172734 .

28. World Health Organization (2009). WHO guidelineson hand hygiene in health care. First Global Patient Safety Challenge. Clean Care is SaferCare. Geneva: WHO.

29. Zapka, C; et al (2017). Comparison of Standard Culture-Based Method to Culture-Independent Method for Evaluation of Hygiene Effects on the Hand Microbiome. mBio. 8(2): e00093-17 